\section{Abnormalities of the Osseous Structures of the Hip and Peri-Articular Soft Tissues}

MRI is the most accurate imaging technique for the detection and assessment of a large number of disorders arising in the bones of the hip or the surrounding soft tissues including osteonecrosis, transient osteopenia, fractures, soft tissue injuries, and tumors. All of these disorders can be readily assessed without the use of a contrast agent. The evaluation of intra-articular structures, in particular the acetabular labrum, however, is probably best accomplished with the use of the direct injection of a Gd-chelate contrast agent into the hip joint (which is discussed in UNIT A26.2).

The protocol given below is utilized on a 1.5-Tesla system for the evaluation of disease in the region of the hip. Some modifications must be made when it is used on low-field $(0.23$ to $0.3 \mathrm{~T})$ systems as will be discussed below.

\section{MR IMAGING OF THE HIPS}

Although MR imaging of the hips for any of these disorders can be satisfactorily performed with either the whole body radiofrequency (RF) coil or a dedicated surface coil, the torso-array coil is generally used. Following the acquisition of a scout sequence in the transverse plane, the first two sequences used are STIR (short tau inversion recovery) and short $T_{\mathrm{E}}$ spin echo sequences of both hips obtained in the coronal or oblique coronal plane. Although a fat-saturated, long $T_{\mathrm{R}} / T_{\mathrm{E}}$ fast (or turbo) spin echo (FSE) sequence can be substituted for the STIR sequence on high-field systems, it appears that the STIR sequence is slightly more sensitive to the presence of abnormalities within the marrow or the surrounding soft tissues and should be used. Only moderate size acquisition matrices (e.g., 192 by 256) are employed. In the authors' experience, the use of higher resolution matrices (e.g., 384 by 512) does not generally aid in the assessment of most disorders and prolongs study time significantly. These are followed by short $T_{\mathrm{E}}$ spin echo and fat saturated, long $T_{\mathrm{E}}$ fast-spin echo sequences obtained in the transverse plane. For the detection and assessment of disease in general, these sequences are the most important and will generally suffice. In those cases in which osteonecrosis $(\mathrm{ON})$ is seen in the femoral head on the coronal sequences, a fat-saturated intermediate $T_{\mathrm{E}}$ sagittal FSE sequence is acquired to help estimate the size of the focus and to look for signs of cortical collapse. The authors also evaluate the articular cartilage and acetabular labrum with this sequence, although its accuracy for these purposes in the absence of intra-articular contrast agent is unknown. On a high-field system with a torso array or similar surface coil in place, the sagittal sequence is performed at much higher resolution than the previously described coronal and transverse sequences. On a low-field system or when a torso array coil has not been applied, the resolution chosen for the sagittal series is the same as that used for the prior series.

On high-field systems $(>1.0 \mathrm{~T})$, this entire protocol will require $\sim 30$ min to perform.

Table A26.1.1 lists the hardware necessary to perform the procedure, along with appropriate parameters. The available gradient strength will depend on the scanner, and the echo times given below should be varied accordingly (the smaller the gradient strength, the longer the echo time for a particular scan).

NOTE: Be sure that technologists and nurses have immediate access to any emergency equipment that may be relevant to a given study, or that may be needed for a particular patient, such as crash carts or oxygen.
BASIC

PROTOCOL 
Table A26.1.1 Equipment Parameters for MRI of the Hips

\begin{tabular}{ll}
\hline Coil type & Torso array coil \\
Gradient coil strength & $25 \mathrm{mT} / \mathrm{m}$ (or whatever the system permits) \\
Cardiac gating & No \\
Peripheral gating & For safety only \\
Respiratory gating & No \\
Respirator & If required by patient \\
Oxygen & If required by patient \\
Motion cushions & Useful \\
Use of contrast agents & No \\
\hline
\end{tabular}

\section{Set up patient and equipment}

1. Interview (screen) the patient to ensure that he or she has no contraindications such as cardiac pacemakers or other implants containing ferromagnetic materials. Also be sure to find out if the patient has any health conditions that may require the presence of special emergency equipment during the scanning procedure, or necessitate any other precautions. The patient should be questioned as to the location of the pain, in particular the side and whether the pain is actually in the hip or, e.g., lower in the thigh.

Generally, standard screening forms (APPENDIX 1) are used for all patients scanned in a magnetic resonance system.

The presence of any ferromagnetic metals may be a health hazard to the patient when he or she is inside the magnet, and will also affect the imaging. If in doubt as to the exact composition of the items, it is best to exclude patients with any metal implants; see Shellock (1996) for discussion of what implants may be safely scanned using magnetic resonance.

Patients may be accompanied into the magnet room by a friend or family member, who can sit in the room during the scan and comfort the patient as needed. This companion must be screened as if they were to undergo MR imaging themselves to ensure the absence of loose metal objects on the body or clothing.

2. If the procedure is a research protocol, have the patient sign any necessary consent forms.

3. Have the patient remove all jewelry and change into a gown to eliminate any metal that might be found in clothing.

4. Inform the patient about what will occur during the procedure, what he or she will experience while in the magnet, and how to behave, including the following:

a. If earphones or headphones are used to protect the ears from the loud sounds produced by the gradients, the patient will be asked to wear these, but will be able to communicate with you at any time during the imaging.

b. The patient will be given a safety squeeze-bulb or similar equipment to request assistance at any time (demonstrate how this works).

c. For good results the patient should not talk, and should avoid or minimize other movement, during each scan-i.e., as long as the banging sounds continue. Between scans, talking is allowed in most cases, but should be avoided when comparative positional studies are being performed; the patient will be informed when this is the case.

d. Nevertheless, the patient may call out at any time if he or she feels it necessary.

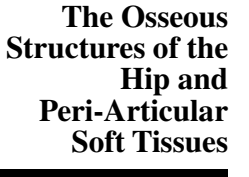

A26.1.2 
5. Have the patient lie down on the table with his or her feet toward the machine. Either before or right after the patient lies down in the supine position, set up any triggering devices or other monitoring equipment that is to be used.

6. Center the patient in a torso array coil or other form of dedicated radiofrequency coil at the hip(s) where the key information is desired. The superior end of the torso array coil should be located at the iliac crest and the inferior end should be well below the greater trochanter.

7. Start to move the patient into the magnet center. Locate the sagittal alignment light along the mid-sagittal plane of the patient. The transverse alignment light should be centered approximately halfway between the anterior/superior iliac spine and the symphysis pubis because the femoral head lies in that location. The coronal alignment light should fall approximately midway between the front and back of the patient. Place him or her into the center of the magnet.

Once this step has been performed, so long as the patient does not move on the table, the table itself can be moved and then replaced in the same position as before without jeopardizing the positioning of one scan relative to another.

8. Sedation is not performed routinely and should be reserved for severe claustrophobia.

\section{Sequence 1: Rapid transverse scout}

9. Obtain a scout series in the transverse plane using the sequence given in Table A26.1.2 (Fig. A26.1.1).

\section{Sequence 2: Coronal fast-STIR}

10. Obtain a coronal or oblique coronal fast-STIR sequence using the parameters given in Table A26.1.3. The plane of the slices should be parallel to an imaginary line joining corresponding locations on the left and right hips on the transverse images (Figs. A26.1.2 and A26.1.3). The field of view (FOV) should be slightly larger than the soft tissues surrounding the hips.

As is true for all multi-slice 2-D techniques, the value of $T_{R}$ should be adjusted depending on the size of the patient. The number of slices is determined by the $T_{R}, T_{E}$, and, in the case of fast-spin echo sequences, the number of echos in the echo train (i.e., echo train length). In the case of $T_{2}$-weighted and STIR sequences, increasing the $T_{R}$ does not alter the contrast and it should be increased so that only a single acquisition will cover the entire volume unless it pushes the acquisition time to the point at which patient motion is likely to interfere with image quality (>10 min).

The authors'system automatically interleaves two or more sequences (doubling or tripling the acquisition time) to cover the appropriate distance if the $T_{R}$ chosen does not permit it to be covered in a single acquisition. For this reason, adjustment of $T_{R}$ while studying the calculated time of acquisition is performed prior to each sequence. The $T_{I}$ will be $\sim 150$ msec at $1.5 \mathrm{~T}$ but should be adjusted as part of the prescan procedure for the sequence.

Note that the appropriate value of $T_{I}$ to null the signal from fat decreases with decreasing field strength and that most systems will slightly adjust the value during the pre-scan to minimize the signal from fat. Choose the value recommended by the equipment manufacturer or the applications specialist.

As with all sequences, the use of a lower field strength magnet may require changes in the sequence parameters (increased FOV or slice thickness, decreased acquisition matrix, greater $N_{a c q}$ or NEX, and others) to compensate for the lower signal-to-noise ratio (SNR). This is particularly true for the STIR sequence, which has a low SNR compared to FSE sequences. 
Table A26.1.2 Transverse Scout

Patient position

Scan type

Imaging plane (orientation)

Central slice or volume center

Echo time $\left(T_{\mathrm{E}}\right)$

Receiver bandwidth (RBW)

Repeat time $\left(T_{\mathrm{R}}\right)$

Flip angle (FA)

Fields of view $\left(\mathrm{FOV}_{\mathrm{x}}, \mathrm{FOV}_{\mathrm{y}}\right)$

Resolution $(\Delta x, \Delta y)$

Number of data points collected $\left(N_{\mathrm{x}}, N_{\mathrm{y}}\right)$

Slice thickness $(\Delta z)$

Number of slices

Slice gap

Number of excitations (NEX)

Number of acquisitions $\left(N_{\text {acq }}\right)$

Swap read and phase encoding

Read direction

Slice locations

Flow compensation

No phase wrap (NPW)

Chemical saturation

Spatial saturation

Scan time
Supine

3-D short $T_{\mathrm{R}}$ gradient echo

Transverse

Halfway between the anterior/superior iliac spine and the symphysis pubis

$1.5 \mathrm{msec}$ (or minimum)

$\pm 32 \mathrm{kHz}$

$6 \mathrm{msec}$ (or minimum)

$30^{\circ}$

$480 \mathrm{~mm}, 480 \mathrm{~mm}$

$1.88 \mathrm{~mm}, 3.75 \mathrm{~mm}$

256,128

$10 \mathrm{~mm}$

$15-20$

$0 \mathrm{~mm}$

2

1

No

Right-left

From above iliac crest to below greater trochanter

No

No

No

No

23-31 sec (depends on size of patient)
The Osseous Structures of the

Hip and

Peri-Articular

Soft Tissues

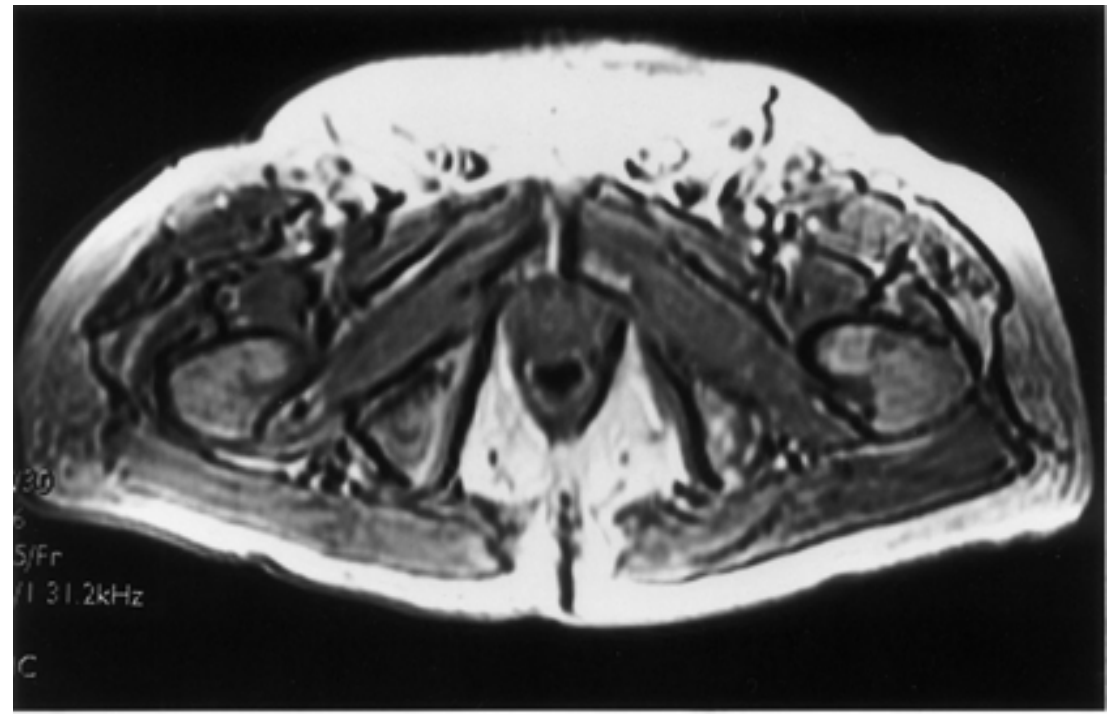

Figure A26.1.1 A representative slice from the transverse scout series obtained using the parameters listed in Table A26.1.2 
Table A26.1.3 Coronal Fast-STIR

\begin{tabular}{ll}
\hline Patient position & Supine \\
Scan type & 2-D inversion recovery fast spin echo \\
Imaging plane (orientation) & Coronal or oblique coronal \\
Central slice or volume center & Through the femoral heads \\
Echo time $\left(T_{\mathrm{E}}\right)$ & $\sim 70 \mathrm{msec}$ \\
Receiver bandwidth (RBW) & $\pm 16 \mathrm{kHz}$ \\
Echo train length (ETL) & 8 \\
Repeat time $\left(T_{\mathrm{R}}\right)$ & $\sim 3500 \mathrm{msec}$ \\
Inversion time $\left(T_{\mathrm{I}}\right)$ & $\sim 150 \mathrm{msec}$ (determined at prescan) \\
Flip angle (FA) & $180^{\circ}$ (or default) \\
Fields of view (FOV, FOV $\mathrm{y})$ & $380 \mathrm{~mm}, 380$ mm (should be chosen to \\
& include soft tissues lateral to \\
& hips- may be larger or smaller) \\
Resolution $(\Delta x, \Delta y)$ & $1.48 \mathrm{~mm}, 1.98 \mathrm{~mm}$ (but depends on \\
& FOV) \\
Number of data points collected $\left(N_{\mathrm{x}}, N_{\mathrm{y}}\right)$ & 256,192 \\
Slice thickness $(\Delta z)$ & $4 \mathrm{~mm}$ \\
Number of slices & $30-40$ (depends on size of patient) \\
Slice gap & $0.5 \mathrm{~mm}$ \\
Number of excitations $(\mathrm{NEX})$ & 2 \\
Number of acquisitions $\left(N_{\mathrm{acq}}\right)$ & 2 \\
Read direction & Superior-inferior \\
Slice locations & From posterior to the SI (sacroiliac) \\
Flow compensation & joints to anterior to symphysis pubis \\
No phase wrap (NPW) & Yes \\
Scan time & Yes \\
& $\sim 7$ min (will depend on factors \\
& discussed in text) \\
\hline
\end{tabular}

\section{Sequence 3: Coronal short $T_{R} / T_{E}$ spin echo}

11. Obtain a coronal or oblique coronal short $T_{\mathrm{R}} / T_{\mathrm{E}} \mathrm{SE}$ sequence using the parameters given in Table A26.1.4 (Fig. A26.1.4). The FOV should be the same as that used for coronal fast-STIR sequence.

The number of slices will be determined by $T_{R}$ and $T_{E} . T_{R}$ can be increased to permit more slices but, if $T_{1}$ weighting is to be maintained, should not be increased $>800$ msec at $1.5 \mathrm{~T}$ (less at lower field strengths). Because of the automatic interleaving of acquisitions, some adjustment of $T_{R}$ with calculated acquisition times is generally performed.

\section{Sequence 4: Transverse short $T_{E}$ spin echo}

12. Use $T_{1}$-weighted coronal images to prescribe transverse images (Fig. A26.1.5). Obtain a short $T_{\mathrm{E}}$ transverse series of images using the parameters given in Table A26.1.5 (Fig. A26.1.6). The FOV should be the same as with above coronal sequences.

In contra-distinction to the $T_{1}$-weighted coronal sequence above, no effort is made to provide $T_{1}$ weighting, so that $T_{R}$ is adjusted to whatever length is required to image the region of interest in one acquisition as discussed in the steps above. The phase encoding and read directions are "swapped" so that the phase ghosts from the anterior wall are projected in the mediolateral direction to avoid hips. 


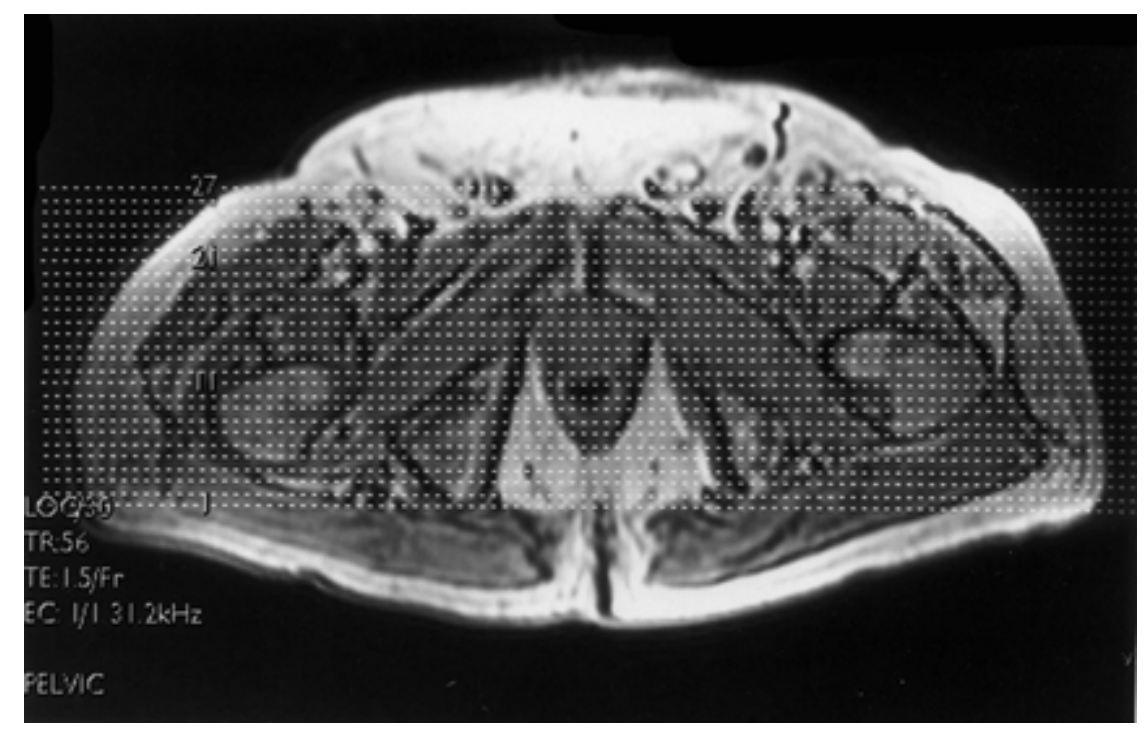

Figure A26.1.2 An image from transverse scout series with locations for the images of both the coronal series (sequences 2 and 3 ) indicated.

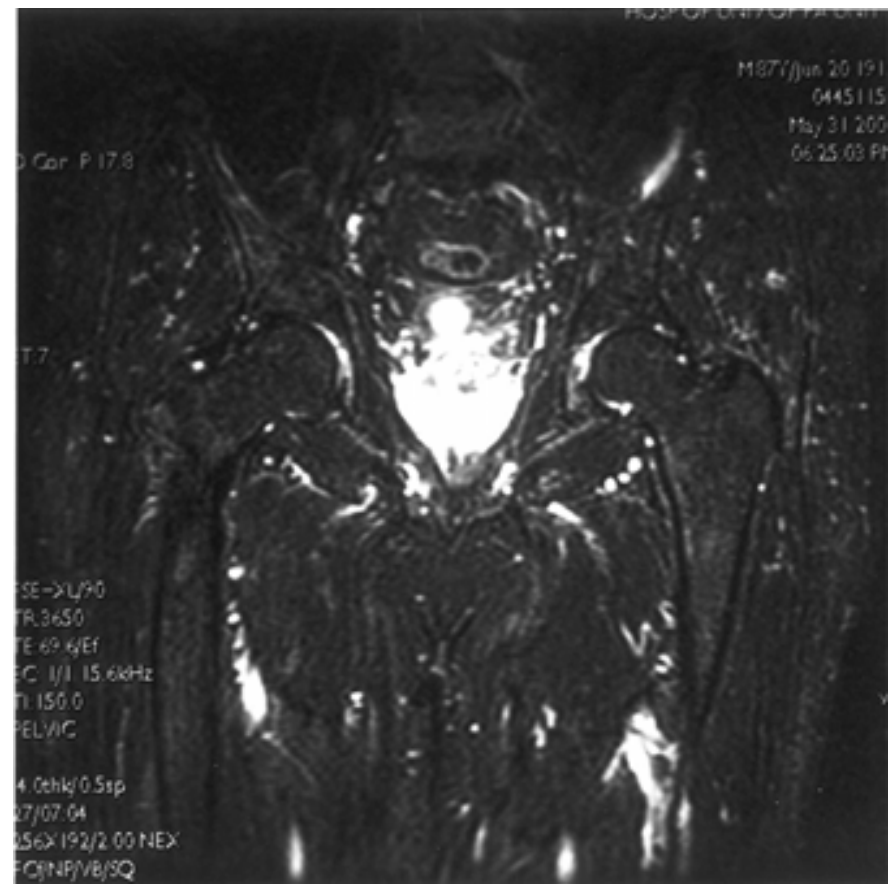

Figure A26.1.3 A representative coronal fast-STIR image obtained with parameters listed in Table A26.1.3.

The Osseous Structures of the

Hip and

Peri-Articular Soft Tissues

A26.1.6 
Table A26.1.4 Coronal $T_{1}$-Weighted Spin Echo

Patient position

Scan type

Imaging plane (orientation)

Central slice or volume center

Echo time $\left(T_{\mathrm{E}}\right)$

Receiver bandwidth (RBW)

Repeat time $\left(T_{\mathrm{R}}\right)$

Flip angle (FA)

Fields of view $\left(\mathrm{FOV}_{\mathrm{x}}, \mathrm{FOV}_{\mathrm{y}}\right)$

Resolution $(\Delta x, \Delta y)$

Number of data points collected $\left(N_{\mathrm{x}}, N_{\mathrm{y}}\right)$

Slice thickness $(\Delta z)$

Number of slices

Slice gap

Number of excitations (NEX)

Number of acquisitions $\left(N_{\text {acq }}\right)$

Read direction

Slice location

No phase wrap (NPW)

Scan time
Supine

Spin echo

Coronal or oblique coronal

Mid-pelvis through lesser trochanter

$8 \mathrm{msec}$ (or minimum)

$\pm 16 \mathrm{kHz}$

$600-800 \mathrm{msec}$ (depending on size of patient)

$90^{\circ}$

$380 \mathrm{~mm}, 380 \mathrm{~mm}$ (should be chosen to include soft tissues lateral to hips - may be larger or smaller)

$1.48 \mathrm{~mm}, 1.98 \mathrm{~mm}$ (depends on FOV)

256, 192

$4 \mathrm{~mm}$

30-40 (depends on size of patient)

$0.5 \mathrm{~mm}$

1

2

Superior-inferior

From posterior to SI joints to anterior to the symphysis pubis

Yes

4-6 min (depends on $T_{\mathrm{R}}, N_{\mathrm{y}}$, and $N_{\text {acq }}$ )

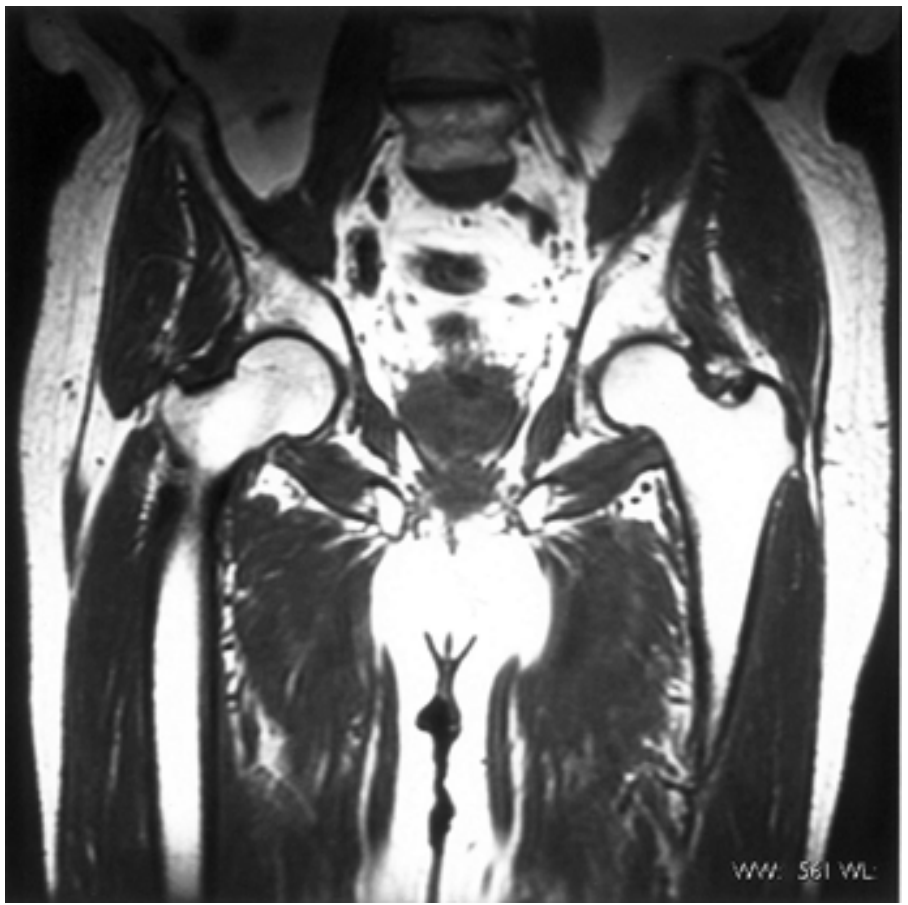

Figure A26.1.4 A representative coronal $T_{1}$-weighted spin echo image obtained with parameters listed in Table A26.1.4. 


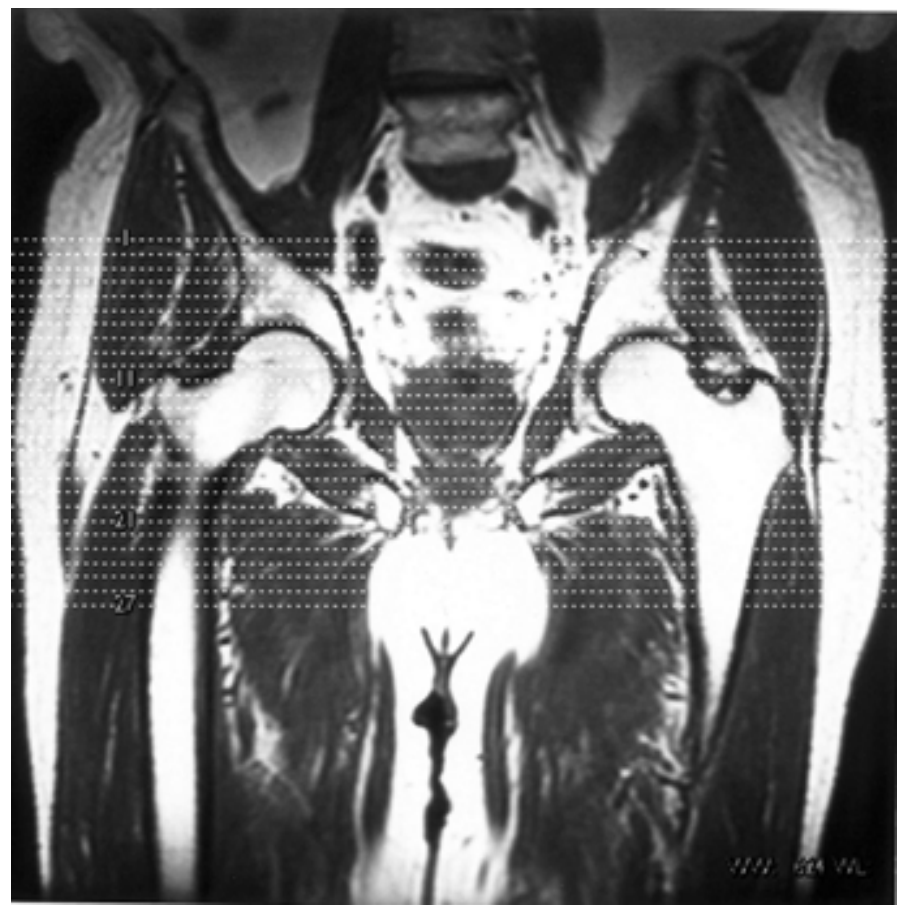

Figure A26.1.5 A $T_{1}$-weighted coronal image with locations of the images from both transverse series (sequences 4 and 5) indicated.

Table A26.1.5 Transverse Short TE Spin Echo

Patient position

Scan type

Imaging plane (orientation)

Central slice or volume center

Echo time $\left(T_{\mathrm{E}}\right)$

Receiver bandwidth (RBW)

Repeat time $\left(T_{\mathrm{R}}\right)$

Flip angle (FA)

Fields of view $\left(\mathrm{FOV}_{\mathrm{x}}, \mathrm{FOV}_{\mathrm{y}}\right)$

Resolution $(\Delta x, \Delta y)$

Number of data points collected $\left(N_{\mathrm{x}}, N_{\mathrm{y}}\right)$

Slice thickness $(\Delta z)$

Number of slices

Slice gap

Number of excitations (NEX)

Number of acquisitions $\left(N_{\text {acq }}\right)$

Swap read and phase encoding

Read direction

Slice locations

No phase wrap (NPW)

Spatial saturation

Scan time
Supine

2-D spin echo

Transverse

Mid-pelvis through lesser trochanter

$8 \mathrm{msec}$

$\pm 16 \mathrm{kHz}$

600-1000 msec (depends on size of patient; no attempt to maintain $T_{1}$ weighting)

$90^{\circ}$

$380 \mathrm{~mm}, 380 \mathrm{~mm}$ (depends on size of patient)

$1.48 \mathrm{~mm}, 1.98 \mathrm{~mm}$ (depends on FOV)

256, 192

$5 \mathrm{~mm}$

30-40 (depends on size of patient)

$1 \mathrm{~mm}$

1

2

Yes

Anterior-posterior

From above iliac crests to below lesser trochanters

Yes

Superior and inferior

4-6 min (depends on $T_{\mathrm{R}}, N_{\mathrm{y}}$, and $N_{\text {acq }}$ ) 


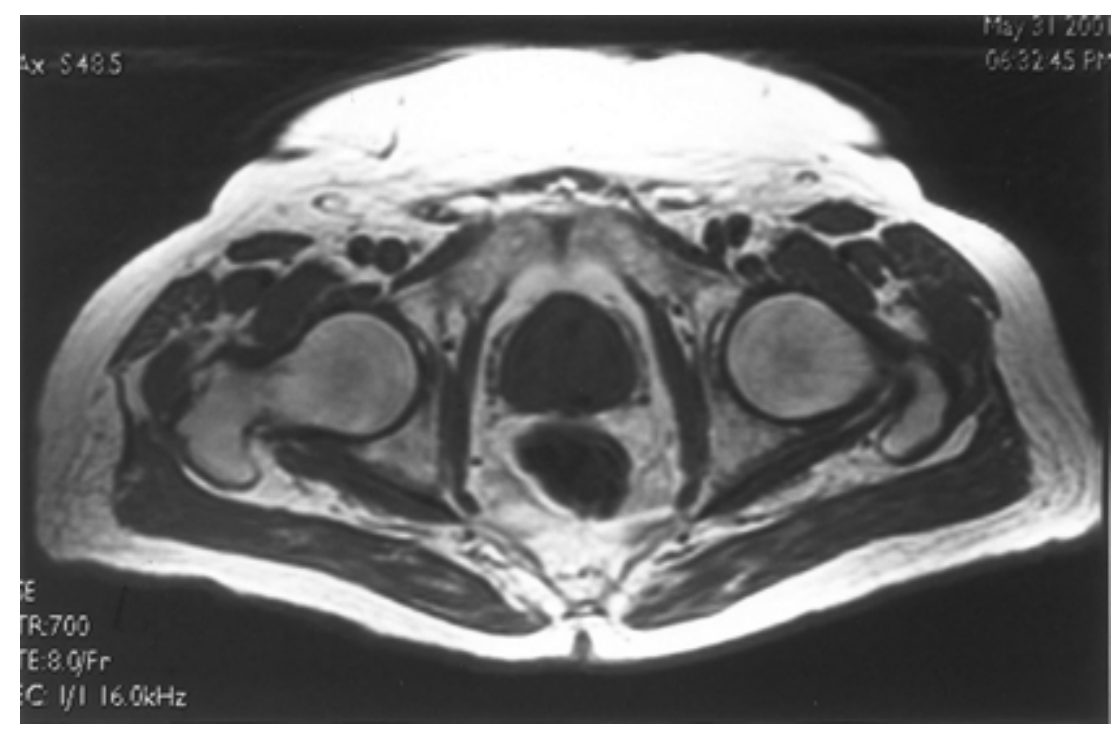

Figure A26.1.6 A representative short $T_{\mathrm{E}}$ spin echo transverse slice obtained using the parameters listed in Table A26.1.5.

Table A26.1.6 Transverse $T_{2}$-Weighted Fat-Saturated Fast-Spin Echo

\begin{tabular}{|c|c|}
\hline Patient position & Supine \\
\hline Scan type & 2-D fast spin echo \\
\hline Imaging plane (orientation) & Transverse \\
\hline Central slice or volume center & $\begin{array}{l}\text { From above iliac crests to below lesser } \\
\text { trochanters }\end{array}$ \\
\hline Echo time $\left(T_{\mathrm{E}}\right)$ & $90 \mathrm{msec}$ \\
\hline Receiver bandwidth (RBW) & $\pm 16 \mathrm{kHz}$ \\
\hline Echo train length (ETL) & 8 \\
\hline Repeat time $\left(T_{\mathrm{R}}\right)$ & $\begin{array}{l}6000 \text { msec (depending on factors } \\
\text { discussed in text) }\end{array}$ \\
\hline Flip angle (FA) & $90^{\circ}$ (or default) \\
\hline Fields of view $\left(\mathrm{FOV}_{\mathrm{x}}, \mathrm{FOV}_{\mathrm{y}}\right)$ & $\begin{array}{l}380 \mathrm{~mm}, 380 \mathrm{~mm} \text { (same FOV as above } \\
\text { sequences) }\end{array}$ \\
\hline Resolution $(\Delta x, \Delta y)$ & $1.48 \mathrm{~mm}, 1.98 \mathrm{~mm}$ \\
\hline Number of data points collected $\left(N_{\mathrm{x}}, N_{\mathrm{y}}\right)$ & 256,192 \\
\hline Slice thickness $(\Delta z)$ & $5 \mathrm{~mm}$ \\
\hline Number of slices & 30-40 (depends on $\left.T_{\mathrm{R}}\right)$ \\
\hline Slice gap & $1 \mathrm{~mm}$ \\
\hline Number of excitations (NEX) & 1 \\
\hline Number of acquisitions $\left(N_{\text {acq }}\right)$ & 2 \\
\hline Swap read and phase encoding & Yes \\
\hline Read direction & Anterior-posterior \\
\hline Slice location & $\begin{array}{l}\text { From above iliac crests to below lesser } \\
\text { trochanters }\end{array}$ \\
\hline No phase wrap (NPW) & Yes \\
\hline Chemical saturation & Yes (fat) \\
\hline Spatial saturation & Yes (superior and inferior) \\
\hline Scan time & $\sim 5 \min \left(\right.$ depends on $\left.T_{\mathrm{R}}\right)$ \\
\hline
\end{tabular}




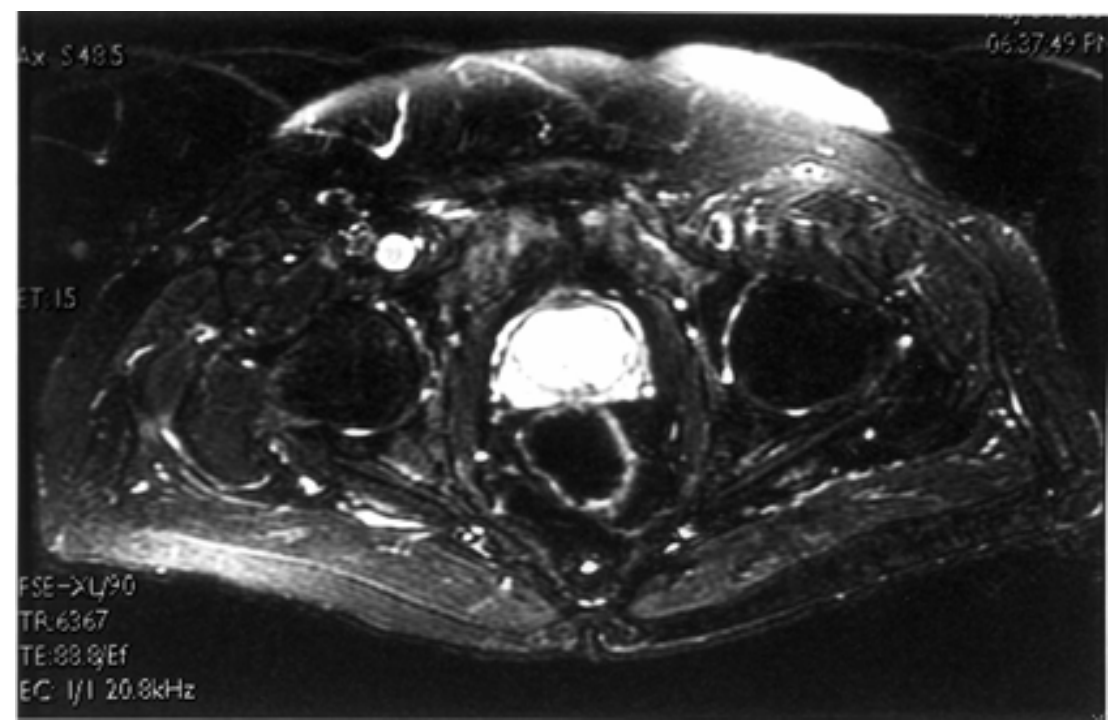

Figure A26.1.7 A representative fat-saturated $T_{2}$-weighted fast-spin echo transverse image obtained with the parameters listed in Table A26.1.6.

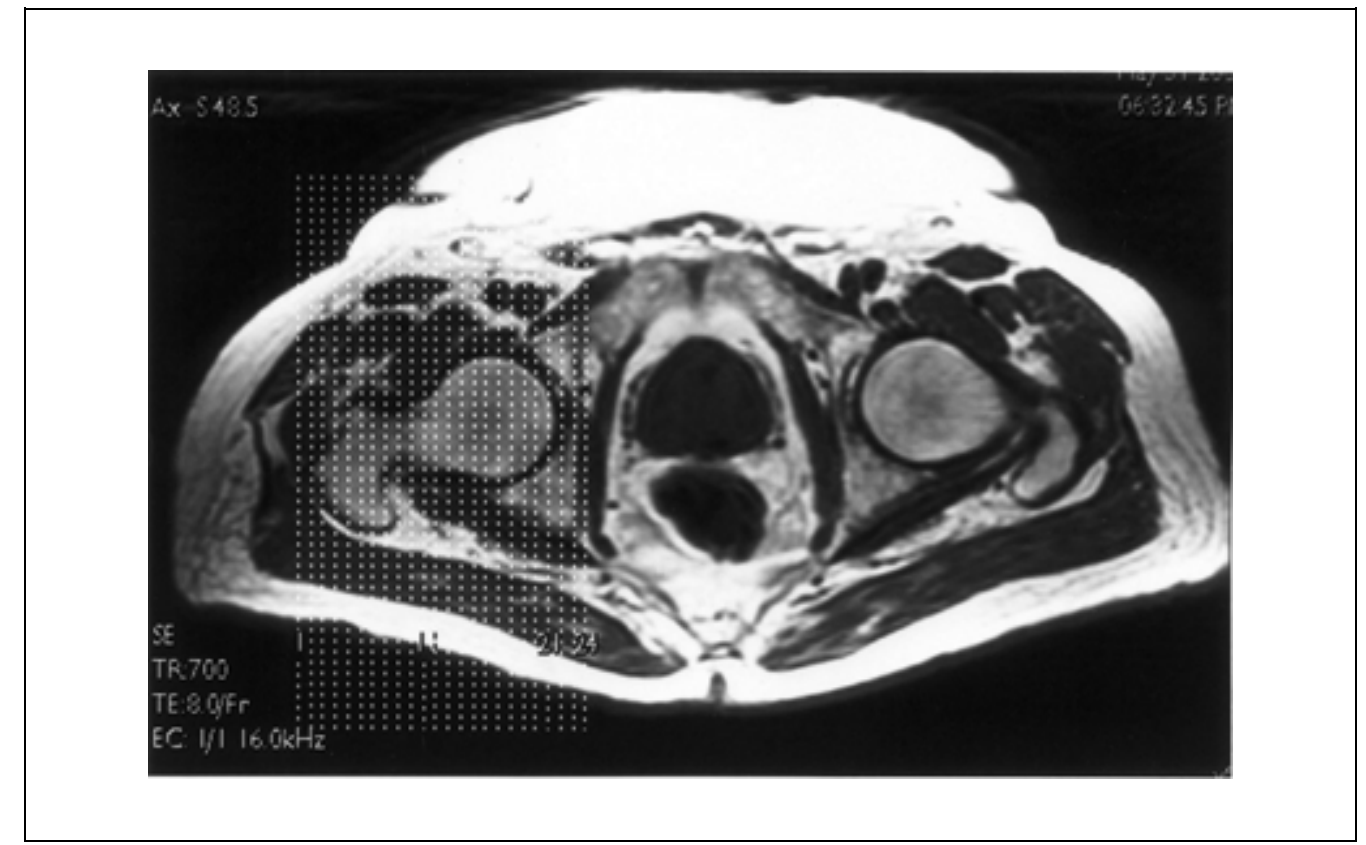

Figure A26.1.8 A short $T_{\mathrm{E}}$ transverse slice with location of the images from the sagittal series indicated.

The Osseous Structures of the

Hip and

Peri-Articular Soft Tissues 
Table A26.1.7 Sagittal Fat-Saturated Intermediate-Weighted FSE

Patient position

Scan type

Imaging plane (orientation)

Central slice or volume center

Echo time $\left(T_{\mathrm{E}}\right)$

Receiver bandwidth (RBW)

Echo train length (ETL)

Repeat time $\left(T_{\mathrm{R}}\right)$

Flip angle (FA)

Fields of view $\left(\mathrm{FOV}_{\mathrm{x}}, \mathrm{FOV}_{\mathrm{y}}\right)$

Resolution $(\Delta x, \Delta y)$

Number of data points collected $\left(N_{\mathrm{x}}, N_{\mathrm{y}}\right)$

Slice thickness $(\Delta z)$

Number of slices

Slice gap

Number of excitations (NEX)

Number of acquisitions $\left(N_{\text {acq }}\right)$

Swap read and phase encoding

Read direction

No phase wrap (NPW)

Chemical saturation

Spatial saturation

Scan time
Supine

Fast spin echo

Sagittal

Through lesser trochanter

$40 \mathrm{msec}$

$\pm 16 \mathrm{kHz}$

8

5000 msec (depends on factors discussed in text)

$90^{\circ}$ (or default)

$200 \mathrm{~mm}, 200 \mathrm{~mm}$

$0.78 \mathrm{~mm}, 1.04 \mathrm{~mm}$

256, 192

$4 \mathrm{~mm}$

20-25

$0.5 \mathrm{~mm}$

3

2

Yes

Anterior-posterior

Yes

Yes (fat)

No

$\sim 5$ min (depends on $T_{\mathrm{R}}$ )

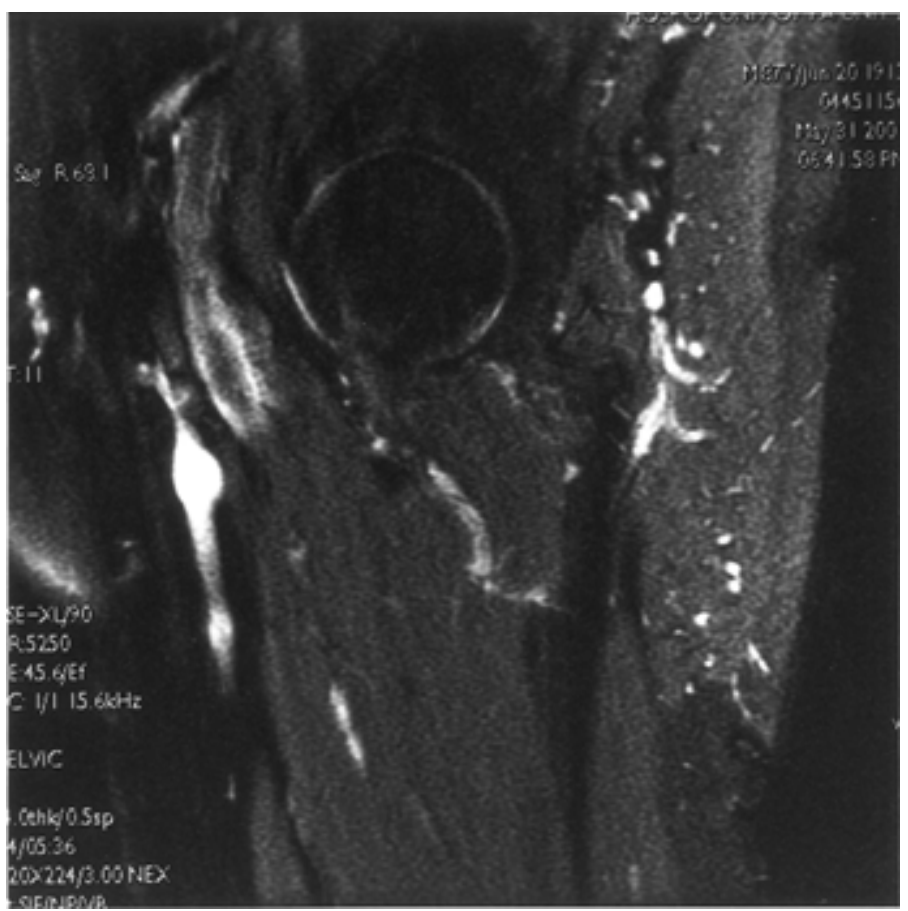

Figure A26.1.9 A representative intermediate weighted fast spin echo image. 
Sequence 5: Transverse fat-saturated long $T_{E} F S E$

13. Run a long $T_{\mathrm{E}}$ FSE sequence using the parameters in Table A26.1.6 (Fig. A26.1.7). The FOV should be the same as with the coronal sequences.

Fat saturation is used because of the relatively bright signal seen from fat with $T_{2}$-weighted FSE sequences.

Selection of precise $T_{R}$ is governed by the same considerations concerning multiple acquisitions discussed above for fast-STIR sequence and will generally be performed at the time of scan.

On low-field systems, fat-saturation cannot be used, thus, the authors typically combine sequences 4 and 5 into one single long $T_{R}$, double spin echo sequence with both short and long $T_{E} s$.

\section{Sequence 6: Sagittal fat-saturated intermediate-weighted FSE (optional)}

14. Use transverse short $T_{\mathrm{E}}$ images to prescribe sagittal images (Fig. A26.1.8). If osteonecrosis is seen in the femoral head on the coronal or transverse images, run a long $T_{\mathrm{R}}$, intermediate $T_{\mathrm{E}}$ FSE sequence using the parameters in Table A26.1.7 (Fig. A26.1.9).

The combination of fat saturation and an intermediate $T_{E}$ makes the articular surface more visible for assessment of contour abnormalities. The selection of $T_{R}$ is determined by the same considerations used for the selection of $T_{R}$ in regard to the fast-STIR coronal and $T_{2}$-weighted fast-spin echo transverse sequences that were discussed above.

For low-field systems, a short $T_{R} / T_{E} S E$ sequence with the same resolution as that used with the other sequences is acquired (see Table A26.1.5 with FOV $V_{x}=F O V_{y}=200 \mathrm{~mm}$ ).

\section{COMMENTARY}

\section{Background Information}

\section{Osteonecrosis and transient osteopenia}

Osteonecrosis (ON) of the femoral head represents the death of bone that occurs in the absence of trauma. Although its etiology is uncertain, there are several well-established pre-disposing factors, of which the most common is the administration of steroids.

In the absence of treatment, most cases of $\mathrm{ON}$ of the femoral head progress to collapse of the cortical surface with the subsequent development of rapidly progressive osteoarthritis. In view of the young age of most patients (30 to 50 ), this poses a major problem because of the limited lifetime of hip prostheses.

Many orthopedists believe that surgical intervention, using such techniques as core decompression when performed before the onset of cortical collapse, can halt progression of the disease. For this reason, there is considerable importance attached to the early diagnosis of ON. MRI is believed to be the best means of establishing the diagnosis prior to collapse.

Transient osteopenia, also known as transient bone marrow edema syndrome, is a poorly understood but self-limited disorder that was originally described in women in the third tri- mester of pregnancy but is more commonly seen in middle-aged and elderly men. The disease is self-limited but can result in a very painful hip for up to 9 months.

\section{Fractures}

Fractures of the hip or pelvis can result from either single traumatic events or from the application of chronic repetitive trauma (stress fractures). Plain films are always the first imaging technique that should be chosen for the detection of fractures but they may fail to detect them. Fractures of the sacrum are most commonly stress fractures and have generally been described in osteoporotic women or in women who have undergone radiation therapy for gynecologic tumors. More recently, they have been identified in female athletes, particularly those who are amenorrheic. More recently, subchondral stress fractures of the femoral head have been described in elderly women that, clinically, may potentially be confused with ON. Although radionuclide bone scans have traditionally been used to detect radiographically occult fractures, most investigators believe that MRI is as sensitive, more specific, and, in older patients, displays earlier positivity than bone scans. 
The early diagnosis of these radiographically occult fractures permits the institution of appropriate therapy at an earlier stage, often avoiding the more aggressive treatment necessary for more advanced fractures.

\section{Soft tissue abnormalities}

Abnormalities of the soft tissues surrounding the pelvis represent a diverse group of disorders. All of these disorders occur elsewhere in the musculoskeletal system and include muscle injury, tendon degeneration, bursitis, and soft tissue tumors. In the authors' experience, the patient's major complaint is pain referring to the hip and the nature of the disorder is often not known. The significance of timely diagnosis of soft tissue abnormalities with MRI is heavily dependent on the nature of the abnormality.

\section{Critical Parameters and Troubleshooting}

The use of MRI for the detection of osseous and peri-articular soft tissue abnormalites is straightforward and accurate studies can be performed on almost any commercial system. The FOV is chosen to include the hips and the adjacent soft tissue that could also give rise to symptoms in the region of the hip. $T_{\mathrm{R}}$ is probably the most critical parameter for each of the sequences and its selection involves the factors that were detailed above for the individual sequences. The use of array coils or other surface coils is not necessary for the MR diagnosis of any of these disorders although occasionally the higher resolution attainable with these coils may be useful to distinguish between two disorders with a similar appearance such as osteonecrosis and the much less common subchondral insufficiency fracture. The added value of obtaining additional images at higher resolution is unproven.

\section{Anticipated Results}

\section{Osteonecrosis and transient osteopenia}

$\mathrm{ON}$ is seen as a well-marginated geographic focus of variable signal in the weight-bearing surface of the femoral head with a well-defined interface. The interface has a low signal on a $T_{1}$-weighted sequence (sequence 3 ; Fig. A26.1.10) and demonstrates parallel high- and low-signal lines on long $T_{\mathrm{E}}$ sequences ("double-line sign"). The region of the femoral head surrounded by the abnormal curvilinear margin most commonly demonstrates the same signal intensity as marrow fat on all sequences. Less commonly, these foci may demonstrate low signal on $T_{1}$-weighted images and high signal on $T_{2}$-weighted images or low signal on both

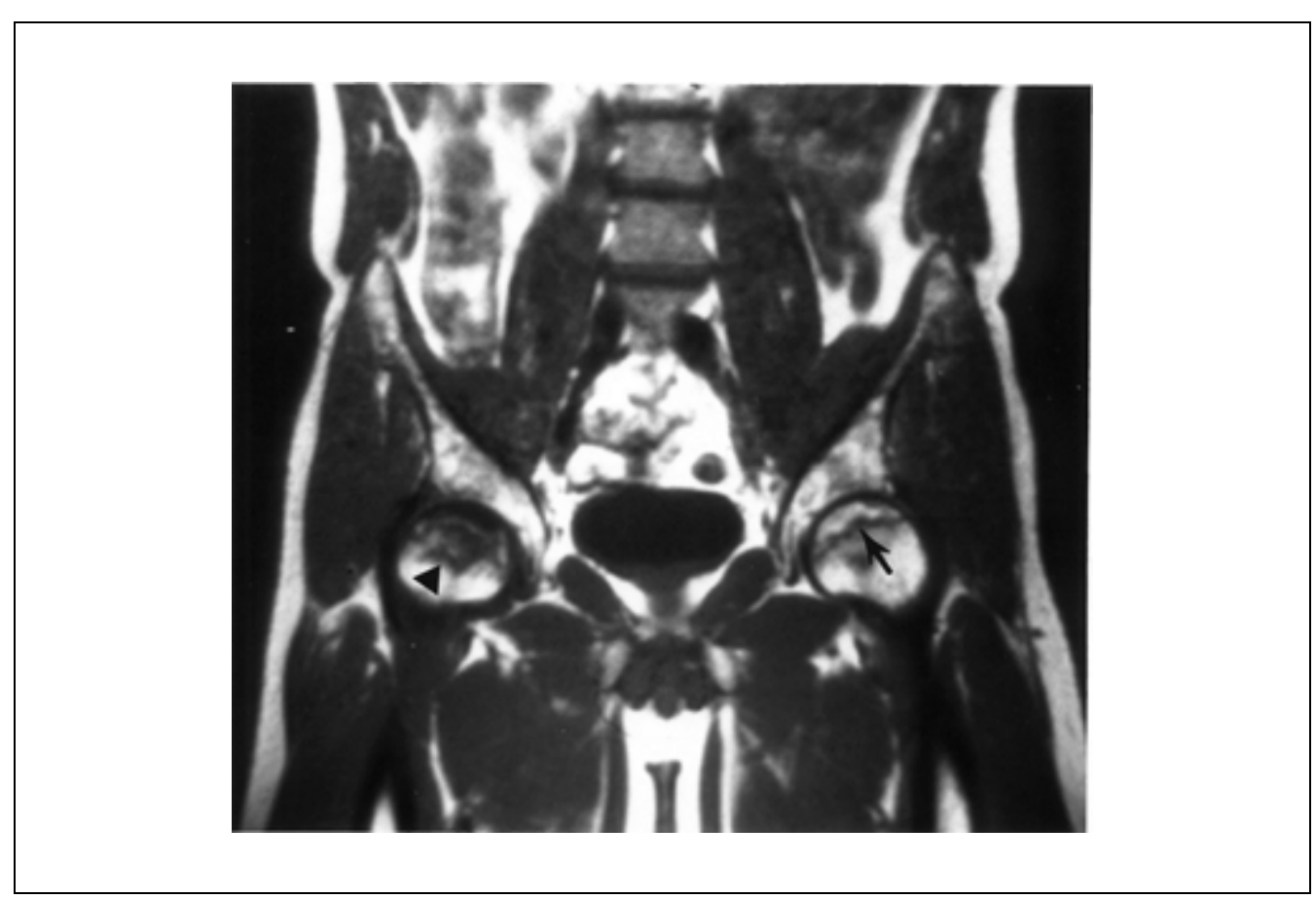

Figure A26.1.10 A coronal $T_{1}$-weighted image through the hips of a patient with bilateral ON. In the patient's left femoral head, a low signal interface surrounds the focus of ON with the signal intensity of fat (arrow). In the right femoral head, the focus of ON demonstrates diffuse low signal (arrowhead). 


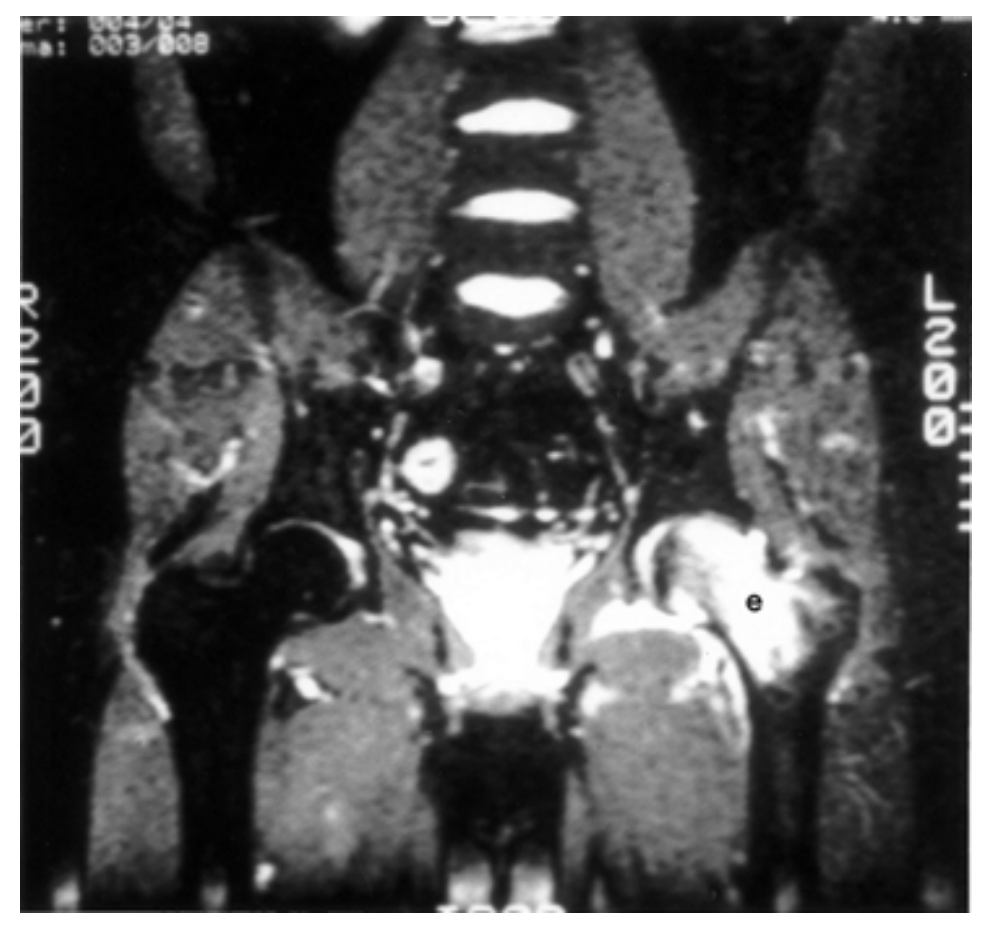

Figure A26.1.11 A fast-STIR coronal image through the hips in a patient with transient bone marrow edema syndrome showing diffuse edema (e) in the left femoral head and neck.

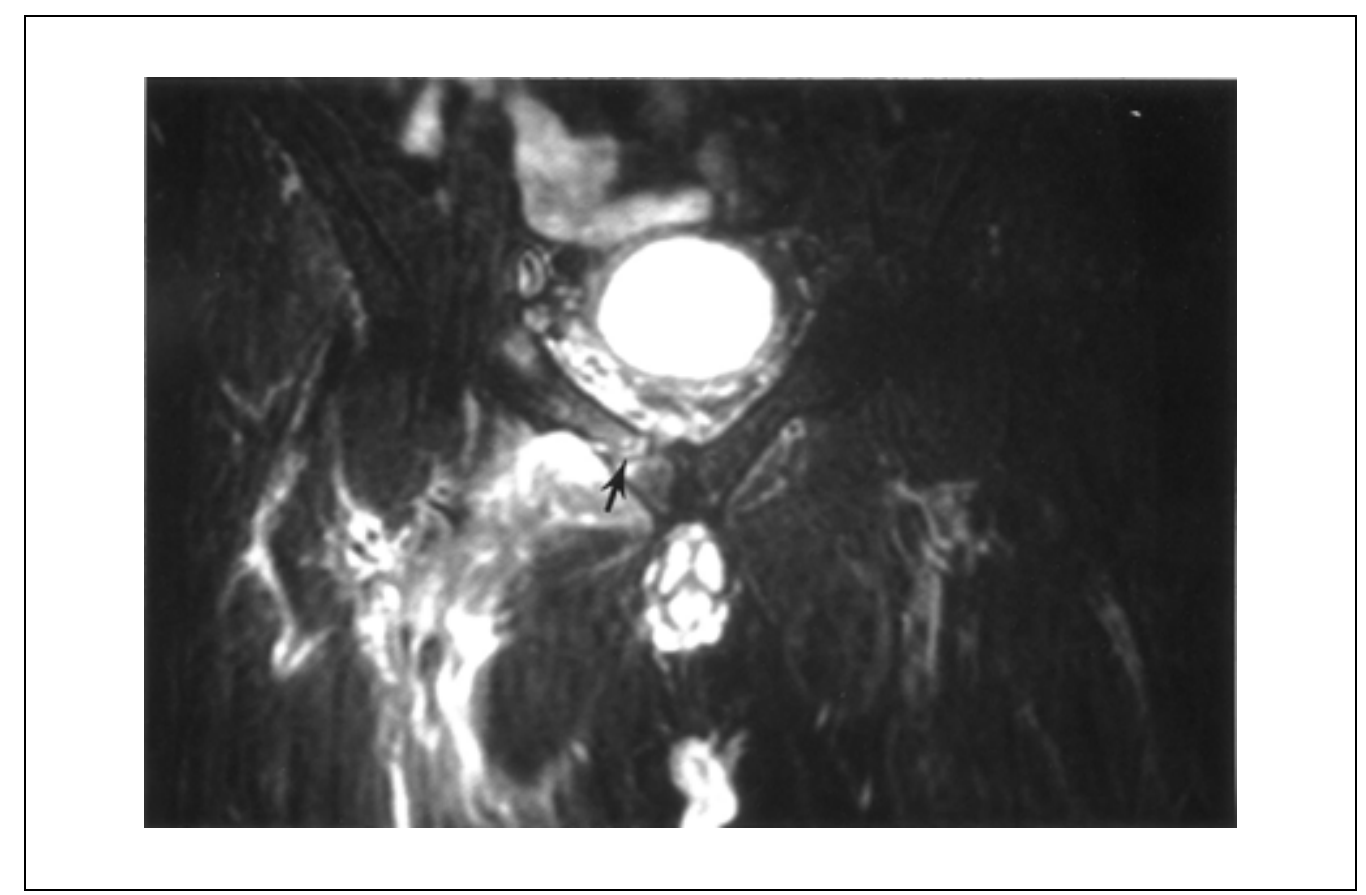

Figure A26.1.12 A fast-STIR coronal image through both ischiopubic rami demonstrates a high-signal fracture (arrow) with edema in the surrounding bone and soft tissue.

The Osseous Structures of the

Hip and

Peri-Articular

Soft Tissues

A26.1.14

Supplement 7 


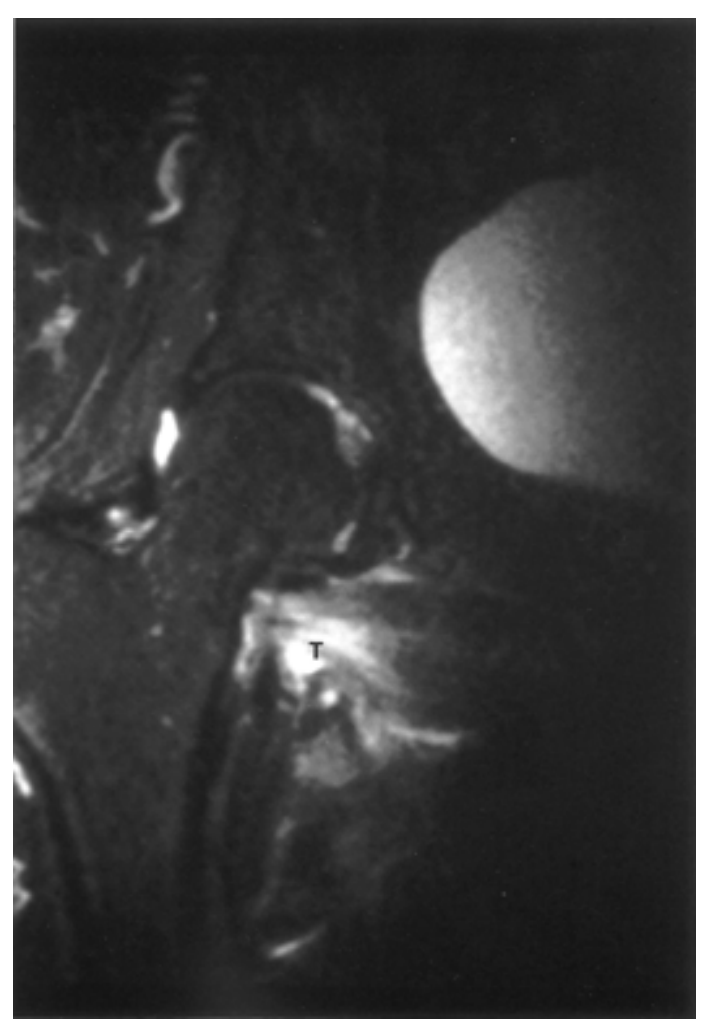

Figure A26.1.13 A coned-down fast-STIR coronal image illustrates the appearance of a tear $(\mathbf{T})$ of the pectineus muscle.

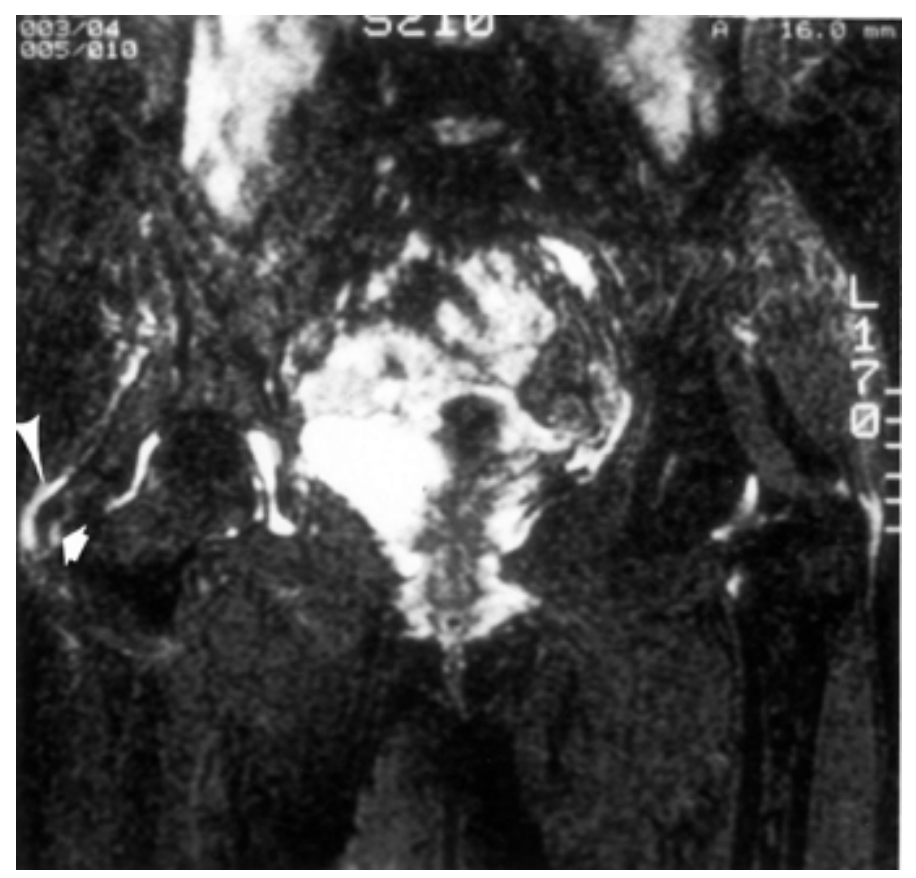

Figure A26.1.14 A fast-STIR coronal image demonstrating a partial tear of the gluteal tendon (arrow) with an accompanying trochanteric bursitis (arrowhead). 
short and long $T_{\mathrm{E}}$ sequences (Mitchell et al., 1987; Lang et al., 1988). In some cases, there is a zone of surrounding bony edema that is seen as diffuse low signal on $T_{1}$-weighted images and high signal on $T_{2}$-weighted images. The presence of bony edema has been claimed by some investigators to be associated with hip pain.

There have been several publications that have investigated the value of measuring the size of the lesion. All have used different techniques with varying degrees of quantitation and all have agreed that the larger the lesion, the greater the probability of collapse even with performance of core decompression surgery (Beltran et al., 1990; Lafforgue et al., 1993). There is no agreement on the best technique to measure size but the percentage (by quartiles) of the weight-bearing surface involved can be readily estimated.

Transient osteopenia is seen as abnormal signal (low on $T_{1}$-weighted and bright on $T_{2^{-}}$ weighted and STIR sequences) that extends throughout the femoral head and neck but without the more discrete focus of abnormal signal with a well-defined margin that is typical of $\mathrm{ON}$ (Fig. A26.1.11; Vande Berge et al., 1999).

\section{Fractures}

Fractures are most commonly seen as linear foci with low signal on all pulse sequences, although in some cases, a high signal linear focus is seen on $T_{2}$-weighted images (Fig. A26.1.12). There is generally ill-defined, abnormal signal in the surrounding marrow (low signal on short $T_{\mathrm{E}}$ sequences, high signal on long $T_{\mathrm{E}}$ and STIR sequences) that is believed to represent marrow edema. With the use of the STIR sequence, the marrow edema may be very bright and obscure the fracture line (Quinn and McCarthy, 1993). When the fracture occurs in the subchondral region of the femoral head, it can be difficult to distinguish from $\mathrm{ON}$, although the double-line sign is less commonly seen (Yamamoto et al., 2001).

In addition to identifying the presence of pelvic fractures, MRI can also identify the presence of associated soft tissue injuries such as muscle tears (see below; Bogost et al., 1995).

Although MRI is the best technique for the detection of subtle fractures, CT (computed tomography) with reformatting or surface rendering is considered to be the most accurate technique for determining the spatial relationship among displaced fragments and for classifying complex acetabular fractures.

\section{Soft tissue abnormalities}

In view of the wide range of possible diagnoses, it is not possible to discuss the MR appearance of every disorder. Benign and malignant soft tissue tumors have a wide variety of appearances that depend on the nature of the tumor (Sundaram et al., 1988). Muscle injury is seen as increased signal on $T_{2}$-weighted or STIR sequences with varying degrees of disruption of muscle architecture (DeSmet and Best, 2000; Fig. A26.1.13). Degeneration and tears of the gluteal tendons are seen as high signal on $T_{2}$-weighted and STIR images within or completely interrupting the normal low signal of the tendons (Kingzett-Taylor et al., 1999; Fig. A26.1.14). Bursitis (iliopsoas, trochanteric) is seen as homogeneous high signal on $T_{2}$-weighted and STIR sequences in the appropriate location. Trochanteric bursitis often accompanies gluteal tendon degeneration but may also occur in its absence (Fig. A26.1.14). The significance of bursitis must be interpreted in light of its location and clinical symptoms.

\section{Literature Cited}

Beltran, J., Knight, C.T., Zuelzer, W.A., Morgan, J.P., Shwendeman, L.J., Chandnani, V.P., Mosure, J.C., and Shaffer, P.B. 1990. Core decompression for avascular necrosis of the femoral head: Correlation between long-term results and pre-operative MR staging. Radiology 175:533536.

Bogost, G.A., Lizerbram, E.K., and Crues, J.V. 3rd. 1995. MR imaging in evaluation of suspected hip fracture: Frequency of unsuspected bone and soft-tissue injury. Radiology 197:263-267.

DeSmet, A.A. and Best, T.M. 2000. MR imaging of the distribution and location of acute hamstring injuries in athletes. A.J.R. 174:393-399.

Kingzett-Taylor, A., Tirman, P.F., Feller, J., McGann, W., Prieto, V., Wischer, T., Cameron, J.A., Cvitanic, O., and Genant, H.K. 1999. Tendinosis and tears of the gluteus medius and minimus muscles as a cause of hip pain: MR imaging findings. A.J.R. 173:1123-1126.

Lafforgue, P., Dahan, E., Chagnaud, C., Schiano, A., Kasbarian, M., and Acquaviva, P.C. 1993. Earlystage avascular necrosis of the femoral head: MR imaging for prognosis in 31 cases with at least 2 years of follow-up. Radiology 187:199-204.

Lang, P., Jergesen, H.E., Moseley, M.E., Block, J.E., Chafetz, N.I., and Genant, H.K. 1988. Avascular necrosis of the femoral head: High-field-strength MR imaging with histologic correlation. Radiology 169:517-524.

Mitchell, D.G., Rao, V.M., Dalinka, M.K., Spritzer, C.E., Alavi, A., Steinberg, M.E., Fallon, M., and Kressel, H.Y. 1987. Femoral head avascular necrosis: Correlation of MR imaging, radiographic staging, radionuclide imaging, and clinical findings. Radiology 162:709-715. 
Quinn, S.F. and McCarthy, J.L. 1993. Prospective evaluation of patients with suspected hip fracture and indeterminate radiographs: Use of $T_{1}$ weighted MR images. Radiology 187:469-471.

Shellock, F.G. 1996. Pocket Guide to MR Procedures and Metallic Objects. Lippincott-Raven, Philadelphia.

Sundaram, M., McGuire, M.H., and Herbold, D.R. 1988. Magnetic resonance imaging of soft tissue masses: An evaluation of 53 histologically proven tumors. Magn. Reson. Imaging 6:237248.

Vande Berge, B.C., Malghem, J.J., Lecouvet, F.E., Jamart, J., and Maldague, B.E. 1999. Idiopathic bone marrow edema lesions of the femoral head: Predictive value of MR imaging findings. Radiology 212:527-535.

Yamamoto, T., Schneider, R., and Bullough, P.G. 2001. Subchondral insufficiency fracture of the femoral head: Histopathologic correlation with MRI. Skeletal Radiol. 30:247-254.

Contributed by J. Bruce Kneeland, Carolyn Kaut Roth, James Garrison, and Anthony Testa

University of Pennsylvania

Philadelphia, Pennsylvania 